\title{
An epistemic bridge for presupposition projection in questions*
}

\author{
Nadine Theiler \\ University of Connecticut
}

\begin{abstract}
It is often assumed that presuppositions in wh-questions project universally. However, Schwarz \& Simonenko (2018) note examples of such questions where universal projection is absent. I discuss their account and propose an alternative: by reasoning about the pragmatics of question-answer discourse, I arrive at a version of Stalnaker's bridge principle that is sensitive to what the speaker already knows about the true answer to the question. This Epistemic Bridge predicts universal projection for canonical information-seeking questions, but less than universal projection for certain non-canonical question uses such as quiz questions.
\end{abstract}

Keywords: presupposition projection, questions, bridge principle, non-canonical questions

The projection behavior of presuppositions in quantified contexts has been studied extensively. Judgments reported in the literature are controversial, and empirical results indicate a large degree of speaker variation (e.g., Chemla 2009, Zehr, Cory Bill, Lyn Tieu, Jacopo Romoli \& Florian Schwarz 2016). It is contested, for example, whether a sentence like (1), with a trigger in the scope of none, has a universal presupposition like (1a) or an existential one like (1b). ${ }^{1}$

(1) None of the participants stopped eating meat during the study.

a. All of the participants used to eat meat. (universal projection)

b. Some of the participants used to eat meat. (existential projection)

This paper develops a theory about projection from the scope of a less studied quantificational construction, namely $w h$-interrogatives. It is often assumed that presuppositions in wh-interrogatives project universally (Schlenker 2008; Abrusán 2014; Nicolae 2015). Motivation comes from judgments like (2), where the factive presupposition is felt to project to the entire wh-domain.

(2) Who among these ten people does Mary regret that Bill invited?

$\rightsquigarrow$ Bill invited all ten of these people.

(Abrusán 2014: 61)

* Thanks to Mike Deigan, Polly Jacobson, Magda Kaufmann, Bernhard Schwarz, Judith Tonhauser, and audiences at SALT30 and the UConn Meaning Group for helpful comments and discussion.

1 I will adopt the convention of underlining presupposition triggers. In (1), e.g., the trigger is stopped. 
An epistemic bridge for presupposition projection in questions

In recent work, however, Schwarz \& Simonenko (2018) show that projection in whinterrogatives depends on the pragmatics of the question use. Certain pragmatically non-canonical questions reliably exhibit projection patterns weaker than universal projection. We will see the same kind of pragmatic variability when examining projection from other kinds of interrogatives and from indefinites and disjunctions in declaratives. To account for projection in all of these environments, I will propose a generalized version of Stalnaker's bridge principle.

The paper is structured as follows. $§ 1$ introduces Schwarz \& Simonenko (2018)'s data, and $\S 2$ discusses their account. $\S 3$ develops the positive proposal, and $\S 4$ presents the predictions this proposal makes. $\$ 5$ shows that the account can be generalized to apply to both declaratives and interrogatives. $\$ 6$ briefly discusses the complications introduced by soft presupposition triggers. $\S 7$ concludes.

\section{Data}

\subsection{Non-universal projection in non-canonical questions}

If a $w h$-interrogative is used as a canonical information-seeking question, presuppositions seem to project universally from its scope (Schlenker 2008; Abrusán 2014; Nicolae 2015). For example, due to the gendered pronouns in (3), the question presupposes that all five zebras are female (see Sudo 2012 for arguments that female gendered pronouns are presuppositional). ${ }^{2}$

(3) Which of our five zebras let herself out of her enclosure? $\rightsquigarrow$ All five zebras are female.

Schwarz \& Simonenko (2018) note several examples of wh-questions where universal projection is absent. These examples can be organized into three categories.

The first category are so-called reference questions (cf. Bolinger 1957; Pope 1976; Rando 1980; Bartels 1999). A reference question, such as B's utterance in (4), is a question asking the addressee to identify a discourse referent that has

2 Some speakers of English have reported not getting a gender inference from she/her pronouns because they have adopted the convention of using these pronouns in a gender-neutral way.

It seems to me that speakers will often adopt other interlocutors' conventions and that they generally try to keep conventions consistent within the same discourse. E.g., if a speaker utters (i), then they make it clear that they use they/their as a gender-neutral pronoun. If this same speaker later on asks (3), we can reasonably conclude that she/her introduces a gender presuppositions.

(i) Zebras are so clever! This one has taught themself to open the food bucket with their hooves.

Readers who have difficulties accessing the intended readings are asked to assume that the relevant example is part of a discourse in which the speaker has earlier made a "convention-establishing" utterance like (i). Thanks to Magda Kaufmann (p.c.) for suggesting this strategy. 
been introduced by an indefinite in the preceding assertion. B's question in (4), unlike the string-identical (3), doesn't presuppose that all five zebras are female. That is, the presupposition projects less than universally.

A: One of our five zebras let herself out of her enclosure.

B: Oh! Which of our five zebras let herself out of her enclosure?

$\varkappa \rightarrow$ All five zebras are female.

The second category are quiz questions such as (5), which doesn't presuppose that all five zebras are female either.

(5) Quiz master: Which of the five zebras at Franklin Park Zoo gained fame last year by letting herself out of her enclosure and roaming downtown Boston? $\leftrightarrow \rightarrow$ All five zebras are female.

I will refer to Schwarz \& Simonenko's third category as too-big-domain questions, as the characteristic property of these questions is that the speaker includes individuals in the wh-domain that aren't possible answers to the question. Schwarz \& Simonenko's example is given in (6), where B's question indeed doesn't have a universal presupposition. More generally, though, we will see that too-big-domain questions don't show non-universal projection as robustly as the other categories.

(6) [It's common knowledge who the 20 players in the team are. It's also commonly known who the three players are that scored goals in the last game.]

A: Crazy Fred is turning into a real problem. Whenever he finds out that one of our players scored a goal in a league game, within a week or two he sends that player a threatening text message.

B: We need to protect our players! Which of them does Fred know scored in the last game? $\not \ngtr$ All 20 players scored.

In addition to Schwarz \& Simonenko's examples, we also find that universal projection is absent in two other kinds of questions, namely rhetorical questions like (7) and echo questions like (8).

(7) You should stop saying that Bo gets along well with the other animals! After all, which of our five zebras threw herself at poor Cem like a maniac?

$\not \rightarrow$ All five zebras are female.

(8) A: There's been a lot of aggression between the zebras recently. Just yesterday I saw Bo throw herself at poor Cem!

B: You saw who throw herself at Cem?

y $\rightarrow$ All zebras are female. 
An epistemic bridge for presupposition projection in questions

\subsection{Confounds to watch out for}

As mentioned initially, judgments about projection in quantified contexts are controversial. What might be behind the disagreement, at least in part, are two confounding processes that can interfere with projection under quantifiers, namely local accommodation and implicit domain restriction.

To see this, let's again consider (9). At least for some speakers this sentence has non-universal projection readings on which it either has no presupposition at all or merely presupposes that some participants used to eat meat. Even if we assume universal projection as the default projection mechanism, we can explain these readings.

We can capture the presupposition-less reading by appealing to local accommodation, a process by which the presupposition becomes part of the at-issue content (Heim 1983). After locally accommodating the presupposition of stopped, (9) would be understood as in (9a).

The existential projection reading can be explained by assuming that the whdomain in (9) is implicitly restricted to only those individuals satisfying the presupposition. That is, (9) would be understood as (9b), where the wh-domain contains only those participants who used to eat meat. If the presupposition projects universally w.r.t. this restricted domain, this only amounts to existential projection w.r.t. the domain of all participants and might hence be felt as existential projection.

(9) None of the participants stopped eating meat during the study.

a. Local accommodation reading: None of the participants are such that they used to eat meat and stopped eating meat.

b. Domain restricted reading: None of the participants that used to eat meat stopped eating meat.

For the purposes of this paper, we are interested in studying the projection properties of $w h$-interrogatives in isolation from these mechanisms. To obtain clearer judgments, we hence want to control for local accommodation and implicit domain restriction as much as possible. In order to prevent local accommodation, we will use hard presupposition triggers, which have been argued not to permit local accommodation (Abusch 2002). Moreover, for probing projection from wh-questions, we are particularly interested in triggers whose presuppositions covary with the whphrase. Triggers that satisfy these criteria and that we will use are female gendered pronouns (Sudo 2012), additives like also (Abusch 2010), and emotive factives like regret (Abbott 2006).

In order to discourage implicit domain restriction as much as possible, we will use partitive wh-phrases of the form which of D num $N$ (e.g., which of our five zebras), as experiments have indicated that these phrases make implicit domain restriction less likely (Geurts \& Bob van Tiel 2016, cf. also Chemla 2009 for French). 
It's worth noting, though, that sometimes it can be unproblematic if an example permits local accommodation or domain restriction. This is because, whenever we are hoping to show that a sentence has a universal projection reading and we do indeed get this reading, it doesn't matter if local accommodation or domain restriction is available, as these mechanisms would prevent universal projection. So the very fact that we do get universal projection shows us that they are not at work.

\subsection{Too-big-domain questions}

Let's return to one of Schwarz \& Simonenko's data points, too-big-domain questions. In their example (6), part of which is repeated in (10), the speaker appears to include the whole sports team in the wh-domain, even though it is known that only some of the players scored goals (and thereby satisfy the factive presupposition).

B: We need to protect our players! Which of them does Fred know scored in the last game?

$\leftrightarrow \rightarrow$ All 20 players scored.

There are two things to note here. One, epistemic factives like know are not generally considered hard triggers and may therefore allow local accommodation. Two, even though the wh-phrase which of them should make implicit domain restriction hard to obtain, it doesn't have the form that has been shown to be effective in preventing implicit restriction. It's thus conceivable that the wh-domain in (11) is understood as further restricted, e.g., to only those players who scored goals. These factors do indeed seem to play a role in (10). If we make the wh-domain fully explicit and use a hard trigger, then the presupposition does seem to project universally:

(11) [Same scenario, but now A and B are scrolling through a list of all players. Currently, there are the names and photos of five players on the screen.]

B: We should think about offering counseling. \#Which of these five players regret that they scored in the last game?

$\rightsquigarrow$ All five players scored.

I conclude that, once we control for local accommodation and domain restriction, too-big-domain questions don't reliably have non-universal projection readings. I will therefore leave these questions aside for the rest of the paper. 
An epistemic bridge for presupposition projection in questions

\section{Discussion of Schwarz \& Simonenko's account}

\subsection{Three felicity conditions}

As we saw in the previous section, presupposition projection in $w h$-questions is affected by the pragmatics of the question use. Schwarz \& Simonenko's account builds on this insight. It assumes three conditions on felicitous canonical question use. These conditions jointly entail universal projection, but can be violated, which, according to Schwarz \& Simonenko, results in non-universal projection.

For example, Schwarz \& Simonenko would say that (12) is felicitous only if the conditions in (13) are fulfilled. It can be shown that, taken together, these conditions entail universal projection (i.e., for (12) they entail that John adopted every zebra).

(12) Which zebra does John regret adopting?

(13) a. Restrictor Homogeneity: The wh-restrictor denotation (here, the denotation of zebra) is constant across the context set $c$.

$$
\forall v, w \in c: \llbracket z e b r a \rrbracket(v)=\llbracket z e b r a \rrbracket(w)
$$

b. No Accommodation: The presuppositions of all answers are either incompatible with the context set or entailed by it. I.e., accommodation is either impossible or unnecessary.

$$
\forall x(c \models \operatorname{zebra}(x) \rightarrow(c \models \neg \operatorname{adopted}(x)(\mathbf{j}) \vee c \models \operatorname{adopted}(x)(\mathbf{j})))
$$

c. Restrictor Economy: The presuppositions of all answers are compatible with the context set.

$$
\forall x(c \mid \operatorname{zebra}(x) \rightarrow c \not \models \neg \operatorname{adopted}(x)(\mathbf{j}))
$$

\subsection{Discussion}

Schwarz \& Simonenko maintain that violating any one of the three conditions will result in the absence of universal projection. I'd like to challenge this claim. As far as I can see, at least two of the conditions, namely No Accommodation and Restrictor Economy, are not necessary for universal projection. Here I show that we can violate them and still get a universal projection reading.

No Accommodation. Recall that the No Accommodation condition requires the presuppositions of all answers to either be incompatible with the context set or entailed by it. In example (14), this condition is violated: the driving test examiner accommodates that all three kids have already failed a driving exam. This information wasn't in the common ground before, nor was it entailed by it. Yet, it seems 
coherent for the examiner to accommodate the universally projected presupposition. Hence, we get universal projection even though No Accommodation is violated.

[Parent talking to driving test examiner after practical exam.]

P: So, which of my three kids also failed their second attempt?

E: Oh, I didn't know it was their second attempt! That's why they were so nervous.

Restrictor Economy. Recall that Restrictor Economy requires the presuppositions of all answers to be compatible with the common ground. We have already seen an example in which this condition gets violated, namely (11), part of which is repeated here in (15). The $w h$-domain contains five players even though it is known that only three players scored in the last game. Yet, the presupposition projects universally, making the utterance infelicitous in the given context.

B: We should think about offering counseling. \#Which of these five players regret that they scored in the last game?

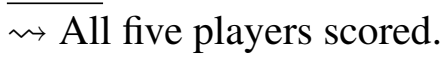

We have thus seen for two of Schwarz \& Simonenko's felicity conditions that they are not necessary for universal projection. This also means that, although the three conditions jointly entail universal projection, we shouldn't think of them as the source (or at least not the only source) of universal projection. The larger aim for the rest of the paper is to spell out what I take to be the source of universal projection in canonical questions - not only in wh-questions, but in questions more generally.

\section{Positive proposal: Epistemic Bridge}

There is a property unifying all the examples of non-universal projection that we have seen so far: the speaker isn't completely ignorant about the question they are asking. They either already know the answer, as is the case with quiz questions and rhetorical questions, or they can exclude at least some of the answers, as we will see is the case with reference questions and echo questions. I will propose a pragmatic account that builds on this observation and tries to elucidate the connection between the felicity of asking a question and the presuppositions of its answers.

\subsection{Previous bridge principles}

In his classic pragmatic account of presuppositions, Stalnaker (1974) famously connected a sentence's presupposition to the felicity conditions of uttering that sentence. This connection was later dubbed Stalnaker's Bridge by von Fintel (2008). 
An epistemic bridge for presupposition projection in questions

Stalnaker's Bridge:

A sentence is felicitous in a context only if its presupposition is entailed by the context set.

While Stalnaker's Bridge can in principle be applied to questions, it doesn't tell us anything directly about the relation between the felicity of asking a question and the presuppositions of its answers. Guerzoni (2003) proposes a bridge principle that does:

\section{Guerzoni's Bridge:}

A question is felicitous in a context only if it can be felicitously answered in the context (i.e., only if the presupposition of at least one of its answers is entailed by the context set).

The problem with (17) is that it only derives existential projection and thus makes wrong predictions for canonical information-seeking questions such as (2) or (3), which reliably exhibit universal projection.

\subsection{The CORE ARGUMENT}

We start with some reasoning about question-answer discourse. I will sometimes refer back to the following argument as the CORE ARGUMENT.

(i) When a speaker asks a question $Q$, they want the addressee to provide the true answer to $Q$ and this answer to be added to the CG.

(ii) With a canonical information-seeking question, the speaker doesn't know which answer is the true complete one.

(iii) Because of (ii), if the speaker wants to guarantee the success of the CG update described in (i), they must make sure that all answers that as far as they know could be the true complete answer are felicitous in the context.

(iv) In particular, following Stalnaker's Bridge, (iii) requires that the presuppositions of all these answers are entailed by the context set.

\subsection{Can true answers be infelicitous?}

Let's spell out more precisely what it is that the speaker seeks to avoid when asking a question. They want to avoid a situation in which the desired CG update can't take place because uttering the true answer is infelicitous.

The following is an example of such as situation. Suppose there are three salient zebras, called A, B and C. Further suppose it's commonly known that only A and C are female, and only B let themself out of their enclosure. 


\section{Context for (19):}

\begin{tabular}{r|lll} 
& A & B & C \\
\hline is female & $\checkmark$ & X & $\checkmark$ \\
let themself out & $\mathbf{X}$ & $\checkmark$ & X
\end{tabular}

Which zebra let herself out of her enclosure?

True answer: B let herself out of her enclosure.

In the given context, the true answer of the question in (19) is B let herself out of her enclosure. The presupposition of this answer, that B is female, is incompatible with the context. This means in particular that it isn't entailed by the context set, which according to Stalnaker's Bridge makes it infelicitous to utter this answer. Global accommodation isn't available as a rescue strategy either, since the presupposition isn't merely not entailed by the context set, but incompatible with it.

Parts of this may sound surprising. Since presuppositions are classically analyzed as entailments, we would expect that an answer can be true at a world only if its presupposition is also true at that world. Switching back to talking about a context set $c$, this means the following: we would expect that there is no such thing as an answer whose presupposition is incompatible with $c$ but whose at-issue content is compatible with $c$ (but that's exactly what I said the true answer to (19) is).

Crucially, however, not all presuppositions are entailments. It has been argued convincingly that the presuppositions of certain triggers like gendered pronouns are not entailed (Sudo 2012) and suggested that the division between entailed and nonentailed presuppositions lines up with the soft/hard distinction (Klinedinst 2016). ${ }^{3}$

We will postpone the discussion of entailed/soft presuppositions until $\S 6$ and first focus on non-entailed/hard presuppositions. With these presuppositions, atissue and presuppositional content are independent. It thus makes sense to distinguish four different cases. Given a sentence $S$ and a context set $c$, it can happen that:

(a) at-issue content and presupposition of $S$ are compatible with $c$,

(b) only at-issue content but not presupposition of $S$ is compatible with $c$,

(c) only presupposition but not at-issue content of $S$ is compatible with $c$,

(d) neither at-issue content nor presupposition of $S$ are compatible with $c$.

The true answer of (19) is an example of (b): going by the at-issue content, it would be a fine utterance in the given context, but its presupposition makes it infelici-

3 While Sudo 2012 and Klinedinst 2016 contain the most explicit discussion of these matters, parallel ideas have been developed in different contexts, especially by Glanzberg (2005), Yablo (2006), and Gajewski (2011). For experimental work on non-entailed presuppositions, see Zehr \& Schwarz 2016, 2018, Creemers, Zehr \& Schwarz 2018, and Schwarz, Djärv \& Zehr 2018. 
An epistemic bridge for presupposition projection in questions

tous. The CORE ARGUMENT in the previous section is based on the assumption that speakers (among other things) avoid asking questions whose true answer falls into category (b).

\subsection{Epistemic Bridge principle}

Recall the conclusion of our CORE ARGUMENT: the presuppositions of all those answers that, as far as the speaker knows, could be true need to be entailed by the context set. In order to make this formally precise, we will first introduce our treatment of questions as well as a few auxiliary notions.

Question meanings will be represented as sets of possible answers, roughly as in a Hamblin/Karttunen account. ${ }^{4}$ Departing from Hamblin/Karttunen, each answer $p$ is modeled not as a simple proposition, but as a pair of propositions $p=\left\langle p_{\alpha}, p_{\pi}\right\rangle$, where $p_{\alpha}$ is the at-issue content of $p$ and $p_{\pi}$ the presupposition of $p$ (following Champollion, Ivano Ciardelli \& Floris Roelofsen 2017). For example, for each of the zebras a, b and c, (20) contains an answer that has as its at-issue content the proposition that the respective zebra escaped and as its presupposition the proposition that that zebra is female.

$$
\begin{aligned}
& \text { 【Which of these three zebras let herself out?』 } \\
& =\{\langle\lambda w \text {.escaped }(w)(\mathbf{a}), \lambda w \text {.female }(w)(\mathbf{a})\rangle, \\
& \langle\lambda w \text {.escaped }(w)(\mathbf{b}), \lambda w \text {.female }(w)(\mathbf{b})\rangle, \\
& \langle\lambda w \text {.escaped }(w)(\mathbf{c}), \lambda w \text {.female }(w)(\mathbf{c})\rangle\}
\end{aligned}
$$

Note that in the spirit of Karttunen \& Peters (1979) and pace Champollion et al. (2017) we don't posit any specific relationship between the at-issue and presuppositional component of the answers. This allows us to represent answers with non-entailed presuppositions, which were discussed in the previous section.

We will further need a way of referring to those answers in a question denotation $Q$ whose at-issue content is compatible with the speaker's belief state $\sigma$. I will write $Q \uparrow \sigma$ for this set, which is defined as follows.

$$
Q\left\lceil\sigma:=\left\{p \in Q \mid p_{\alpha} \cap \sigma \neq \emptyset\right\}\right.
$$

Within this setup, we can now formulate the conclusion of the CORE ARGUMENT as a bridge principle:

4 There are various design choices that don't bear on the current proposal and which are therefore not discussed here. It doesn't matter, e.g., if a question receives a mention-all or mentionsome interpretation-the CORE ARGUMENT goes through for either. Instead of using a Hamblin/Karttunen semantics, we could implement the account in another framework. In $\S 5$ we will in fact sketch an implementation in inquisitive semantics (Ciardelli, Jeroen Groenendijk \& Floris Roelofsen 2018). 


\section{Epistemic Bridge}

A question $Q$ is felicitous relative to a context set $c$ only if the presuppositions of all answers that the speaker considers possibly true are entailed by the context set (i.e., only if $\forall p \in Q \mid \sigma: c \subseteq p_{\pi}$ ).

\section{Predictions}

In this section, we will examine the predictions that Epistemic Bridge makes for wh-questions on their canonical ( $\$ 4.1)$ and non-canonical use ( $\$ 4.2)$, as well as for other interrogative forms such as alternative questions and polar questions (§4.3).

\subsection{Canonical info-seeking $w h$-questions}

If a speaker uses a question $Q$ as a canonical information-seeking question, they don't yet know the answer to $Q$. There are two cases to distinguish: either the speaker is completely ignorant w.r.t. $Q$ or they have some prior knowledge w.r.t. $Q$.

Let's start with the first case. If a speaker is completely ignorant w.r.t. $Q$, all possible answers to $Q$ are compatible with the speaker's belief state. This means, $Q\left\lceil\sigma=Q\right.$. Epistemic Bridge requires that $\forall p \in Q\left\lceil\sigma: c \subseteq p_{\pi}\right.$, which in this case boils down to $\forall p \in Q: c \subseteq p_{\pi}$. This requirement amounts to universal projection: $c$ needs to entail the presuppositions of all answers in $Q$. We hence make the desired prediction that the "default" behavior of presuppositions in $w h$-questions is to project universally. Moreover, since we derived Epistemic Bridge from general pragmatic considerations, we have an explanatory account of this projection pattern.

Let's now assume that the speaker has prior knowledge w.r.t $Q$, i.e., $Q\lceil\sigma \subset Q$. In this case we predict that presuppositions don't project universally but merely project to all members of $Q\lceil\sigma$. This has an interesting consequence: we expect that speakers can deliberately use a hard presupposition trigger to convey their prior knowledge. For example, if the speaker already knows that the zebra in question is female, (23) would be a good choice even in a situation where not all salient zebras are female. This is the case because by uttering (23) in such a situation the speaker implicates that the they have already ruled out all non-female zebras.

(23) [It is common knowledge that not all salient zebras are female.] Which of our zebras let herself out of her enclosure?

This prior-knowledge implicature can be computed as follows.

(i) The speaker used the presupposition triggers herself/her in their question.

(ii) The speaker obeys Epistemic Bridge, i.e., $\forall p \in Q\left\lceil\sigma: c \subseteq p_{\pi}\right.$. 
An epistemic bridge for presupposition projection in questions

(iii) Because of (i), it follows from (ii) that $Q\lceil\sigma$ consists exclusively of answers about female zebras. In other words, the speaker already knows it was a female zebra.

\subsection{Non-canonical $w h$-questions}

Quiz questions. The characteristic property of a quiz question like (24) is that the speaker (but not the hearer) already knows the answer.

Which of the five zebras at Franklin Park Zoo gained fame last year by letting herself out of her enclosure and roaming downtown Boston?

Suppose that $p^{*}$ is the true answer of $Q$. Then the speaker's belief state is compatible with only $p^{*}$, i.e., $Q\left\lceil\sigma=\left\{p^{*}\right\}\right.$. In this case Epistemic Bridge requires only that $c \subseteq p_{\pi}^{*}$, i.e., that the presupposition of $p^{*}$ is entailed by the context set. In other words, only the presupposition of the true answer projects. This is in line with our intuitions for (24).

Rhetorical questions. Rhetorical questions like (25) have been analyzed as questions whose true answer is already part of the common ground (Caponigro \& Sprouse 2007; Biezma \& Rawlins 2017). This means in particular that the speaker knows the true answer. So, as with quiz questions, if $p^{*}$ is the true answer, then $Q\left\lceil\sigma=\left\{p^{*}\right\}\right.$ and Epistemic Bridge requires only the presupposition of $p^{*}$ to project. For instance, the question in (25) makes it clear that the true answer is Bo threw herself at Cem. We hence predict (25) to presuppose only that Bo is female, which is in line with our intuitions.

(25) You should stop saying that Bo gets along well with the other animals! After all, which of our five zebras threw herself at poor Cem like a maniac?

We have seen that with quiz questions and rhetorical questions, Epistemic Bridge correctly predicts less than universal projection. Moreover we make a more specific prediction than just absence of universal projection or existential projection: we predict, and this seems to conform to our intuitions, that it is exactly the presupposition of the true answer that projects.

Reference questions and echo questions. Our examples of reference and echo questions are repeated in (26) and (27). Suppose they are uttered in a context where not all zebras are female and it is common ground which zebras are female. We observe that (26) and (27) are felicitous in such a context. We can hence conclude that the presuppositions in these questions don't project universally-otherwise they 
would clash with the common ground. What we want Epistemic Bridge to predict here is precisely that (26) and (27) are felicitous in the given context.

A: One of our five zebras let herself out of her enclosure.

B: Oh! Which of our five zebras let herself out of her enclosure?

A: I saw Bo throw herself at poor Cem recently!

B: You saw who throw herself at Cem recently?

To begin with, observe that both of these questions inquire about the preceding utterance. We may take the true answer of an echo question to simply be the utterance preceding the question, and a reference question can truthfully be answered by a more specific version of that preceding utterance. For example, the true answer of the echo question in (27) would be I saw Bo throw herself at poor Cem. And assuming that Megan is the zebra who let herself out, the true answer of the reference question in (26) is Megan let herself out. So, even if a speaker asking a reference or echo question doesn't know the true answer of that question, they know that if uttering the preceding assertion was felicitous, then uttering the true answer will be felicitous too.

Now, in both of our examples the questions are preceded by an assertion that uses the same presupposition trigger as the question. In terms of Epistemic Bridge, we can think about this as follows. B knows from A's assertion that it was a female zebra that let herself out/threw herself at Cem. Hence all $p \in Q\lceil\sigma$ are answers whose at-issue content states that a female zebra let herself out/threw herself at Cem. Epistemic Bridge requires $c$ to entail for each $p \in Q\lceil\sigma$ that the zebra which $p$ is about is female. Since it is common ground which zebras are female, this requirement is satisfied and we predict (26) and (27) to be felicitous in the given context, as desired.

\subsection{Other interrogative forms}

Schwarz \& Simonenko's felicity conditions, discussed in $§ 2$, make reference to notions that are specific to $w h$-questions, such as the $w h$-restrictor denotation. Their account thus doesn't carry over to other interrogative forms like polar and alternative interrogatives. By contrast, Epistemic Bridge only uses the general notion of answers, which is shared by all question types. The predictions can therefore straightforwardly be transfered to other interrogative forms: we predict that all interrogatives exhibit universal projection on their canonical information-seeking use, but weaker projection patterns on certain non-canonical uses. 
An epistemic bridge for presupposition projection in questions

Alternative questions. With alternative questions, this prediction is confirmed. As noted by Abenina Adar \& Yael Sharvit (2018), presuppositions project universally in alternative questions. For instance, (28) presupposes that A already has both a canary and a parrot, which means that the presuppositions in both answers project.

A: I adopted another bird! Now there are three of them.

B: Oh, wow! Did you adopt another canary or another parrot?

$\rightsquigarrow$ A already has both a canary and a parrot.

Alternative questions can also be used in quiz or exam contexts. Just as with whquestions, we find non-universal projection patterns in these contexts. For example, if the same interrogative as in (28) is part of a listening comprehension exam, as in (29), we no longer get the inference that Hilda has both a canary and a parrot. That is, it's not the case that the presuppositions of both answers project.

[From a listening comprehension exam:]

In the conversation you just listened to Hilda is worried about the latest addition to her pet family. Did you understand what kind of pets she has?

Did Hilda adopt another canary or another parrot?

$\Varangle \rightarrow$ Hilda already has both a canary and a parrot.

As predicted by Epistemic Bridge, here only the presupposition of the true answer projects. A student taking the exam may therefore use the presupposition to reason about the correct answer: if they know, e.g., that Hilda didn't have a canary yet, they can conclude that the second answer must be the right one.

Polar questions. Testing presupposition projection in polar questions isn't straightforward, since with most triggers, both the positive and the negative answer have the same presupposition. In (30), for example, both answers presuppose that Bo is female. So existential and universal projection would yield the same result.

Did Bo let herself out of her enclosure?

What we need is a presupposition trigger that interacts with negation in such a way that the presupposition of a negated statement is different from that of an affirmative statement. A good candidate for a trigger like this is the scalar focus particle even. Roughly, when appearing in an affirmative assertion, even presupposes that its prejacent is the lowest item on a likelihood scale (e.g., Karttunen \& Karttunen 1977). For instance, in (31) it presupposes that June knowing Finnish is less likely than her knowing another language (plausibly because Finnish is the hardest). 
June even speaks $[\text { Finnish }]_{F}$.

$\rightsquigarrow$ Finnish is the least likely language for June to know.

Under negation, though, this presupposition is reversed. In (32) even presupposes that Finnish is the most likely language for June to know (which means that not knowing it is unexpected).

June doesn't even speak [Finnish $]_{F}$.

$\rightsquigarrow$ Finnish is the most likely language for June to know.

If even appears in a polar question, as in (33), there is a reading under which the presupposition patterns with that under negation (i.e., Finnish is the most likely language). Interestingly, under this reading the question furthermore conveys a negative bias: the speaker expects that June can't speak Finnish (Guerzoni 2003).

Does June even speak [Finnish $]_{F}$ ?

$\rightsquigarrow$ Finnish is the most likely language for June to know.

In a nutshell, this reversal can be captured if (i) we treat even as presupposing that its prejacent is the least likely element among the focus alternatives of the prejacent, and (ii) we assume that even scopes over negation (Karttunen \& Karttunen 1977; Wilkinson 1996; Guerzoni 2003). ${ }^{5}$ Then, in the unnegated (31), even presupposes that June speaking Finnish is less likely than her speaking other languages. In the negated (32), by contrast, even presupposes that June not speaking Finnish is less likely than her not speaking other languages, i.e., that Finnish is the most likely language for her to know.

For even in polar questions, Guerzoni proposes that, at least on one reading, even in the negative answer scopes over negation, just as it would in an assertion. This means each of the two answers of the question has a different presupposition. The positive answer presupposes that Finnish is the least likely language for June to know, whereas the negative answer presupposes that it's the most likely language.

Clearly, these presuppositions are incompatible. Moreover, if it is part of the common ground that Finnish is the most likely language (and this is the context in which the negatively biased reading would arise), then the presupposition of the positive answer will be incompatible with the common ground. Guerzoni uses this fact to derive the bias via the following argument:

"If the speaker decides to formulate a question in a way that, given the context, excludes the possibility of an affirmative answer, he must be biased towards the negative one." $\quad$ (Guerzoni 2003: 80)

5 For competing theories that assume lexical ambiguity rather than scopal ambiguity, see, e.g., Rooth 1985; Rullmann 1997; Giannakidou 2007. 
An epistemic bridge for presupposition projection in questions

Within the Epistemic Bridge account, we can derive the negative bias via essentially the same argument, but are able to formulate it in a more explicit way: ${ }^{6}$

(i) It is part of the common ground that Finnish is the most likely language for June to know.

(ii) Therefore, the presupposition of the positive answer is incompatible with the common ground.

(iii) The speaker obeys Epistemic Bridge, i.e., $\forall p \in Q\left\lceil\sigma: c \subseteq p_{\pi}\right.$.

(iv) But because of (ii), (iii) can't be satisfied if $Q\lceil\sigma$ contains the positive answer.

(v) So it must be that $Q\lceil\sigma$ contains only the negative answer. In other words, the speaker believes the negative answer is true.

Assuming the scope theory of even, Epistemic Bridge thus makes correct predictions about even in polar questions. It predicts that if a context is incompatible with the presupposition of the positive answer, an addressee may resolve this conflict by computing an implicature of negative epistemic bias. This is similar to the prior knowledge implicature discussed in $\$ 4.1 .^{7}$

\section{Extension to declaratives: Inquisitive Epistemic Bridge}

Epistemic Bridge was introduced as a principle for predicting presupposition projection in interrogatives. But there are data points suggesting it can also fruitfully be applied to certain declarative environments, especially disjunctions and indefinites. The projection patterns we find with these constructions closely parallel those we saw with questions: "canonical" disjunctions and indefinites give rise to universal projection, while their "non-canonical" versions exhibit non-universal projection.

What is the relevant sense of canonical in this context? Like questions, disjunctions and indefinites are linked to speaker ignorance. The speaker of (34), for instance, uses a disjunction because they don't know which disjunct is true. Similarly, the speaker of (35) uses an indefinite because they don't know which exact zebra let herself out. I take these ignorance-based uses to be the canonical ones

6 Thanks to Bernhard Schwarz (p.c.) for pointing out that Epistemic Bridge makes the right predictions for even in polar questions.

7 Above we have seen another example, (11), where universal projection is incompatible with the context. There I took this incompatibility to be what makes the question infelicitous. Here on the other hand, I suggested that, rather than causing infelicity, it appears that the incompatibility can get resolved if the addressee computes an epistemic bias implicature. Why is no analogous strategy available for (11)? That is, why can't the conflict there be resolved by computing a prior knowledge implicature? I will leave an explanation of this difference for future work. 
(for indefinites, these uses are also called epistemically non-specific). As indicated in (34) and (35), the presuppositions project universally.

(34) Either Bo let herself out or Sam let herself in (but I don't know which).

$\rightsquigarrow$ Bo is female and Sam is female.

(35) Some zebra let herself out of her enclosure (but I don't know which zebra). $\rightsquigarrow$ All salient zebras are female.

Both indefinites and disjunctions also have non-canonical uses. A speaker can, e.g., use a disjunction in a rhetorical way, as in (36), by choosing one disjunct that is transparently false. Indefinites can be used in an epistemically specific way, as in (37). As indicated in these examples, the presuppositions don't project universally.

Either someone helped Bo escape from her enclosure or I'm the king of France.

$\leftrightarrow \rightarrow$ France has a king.

A certain zebra let herself out of her enclosure. $\not \rightarrow$ All salient zebras are female.

If we formulate Epistemic Bridge in inquisitive semantics (Ciardelli et al. 2018), we can capture these data points. This move involves only one change: instead of talking about answers, Inquisitive Epistemic Bridge in (38) makes reference to socalled resolutions. ${ }^{8}$ In broad strokes, if disjunctions and indefinites are analyzed as alternative-introducing (Kratzer \& Junko Shimoyama 2002, Simons 2005, AlonsoOvalle 2006), each disjunct and each instantiation of the indefinites contributes a resolution. We further assume that for (34) and (35), $P\lceil\sigma=P$, whereas for (36) and (37), $P\lceil\sigma \subset P$. Given this, Inquisitive Epistemic Bridge makes the desired predictions.

(38) Inquisitive Epistemic Bridge

A question or assertion $P$ is felicitous relative to a context set $c$ only if the presuppositions of all resolutions of $P$ that the speaker considers possibly true are entailed by the context set (i.e., only if $\forall p \in P\left\lceil\sigma: c \subseteq p_{\pi}\right.$ ).

\section{What about soft triggers?}

Finally, let's return to soft triggers, which we have been leaving aside for much of the paper. The main data point to note here is that, unlike with hard triggers, we also find non-universal projection in canonical information-seeking questions:

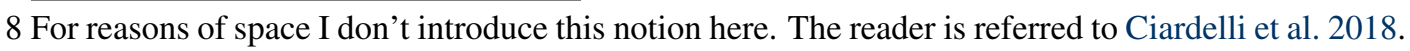


An epistemic bridge for presupposition projection in questions

(39) [Looking for participants for a study on nicotine withdrawal in young adults.] Which of these ten students recently stopped smoking?

$\not \rightarrow$ All ten students used to smoke.

As discussed in $\S 1.2$, this can be explained by appealing to local accommodation, a mechanism that has been argued to be available for soft presupposition but not for hard ones (Abusch 2010). Local accommodation can be captured using an operator (cf. Beaver \& Krahmer 2001) that makes the presupposition of each answer part of the answer's at-issue content. This trivially satisfies Epistemic Bridge.

One intuitive way of thinking about the local accommodation reading of a question is that the presupposition becomes part of what is asked. The speaker leaves it to the addressee to ensure that the presupposition of the true answer is met. This is a useful and natural strategy of inquiry, and unlike with local accommodation under negation, there doesn't seem to be anything "metalinguistic" about local accommodation in questions.

\section{Summary and outlook}

This paper developed an account of presupposition projection in different constructions that involve speaker uncertainty, including $w h$-, polar and alternative questions as well as indefinites and disjunction. For pragmatically canonical versions of these constructions, the proposed analysis predicts universal projection, while for their non-canonical versions it predicts non-universal projection.

The account relies on Epistemic Bridge, a generalized version of Stalnaker's Bridge. Pragmatic bridge principles are attractive because they derive projection from something more basic, namely the process of information exchange. But they have an important limitation: they can't capture projection in complex sentences. The proposed account doesn't aim at replacing compositional theories of presupposition projection, but is intended to work in tandem with them. Epistemic Bridge expects as its "input" a set of propositions, each associated with a presupposition. It thus operates on the output of a compositional theory in a post-compositional way.

Future work will focus on gauging a suitable division of labor between a postcompositional theory of presupposition like Epistemic Bridge and a fine-grained compositional theory such as different dynamic or trivalent accounts.

\section{References}

Abbott, Barbara. 2006. Where have some of the presuppositions gone? In Betty

Birner \& Gregory Ward (eds.), Drawing the Boundaries of Meaning: Neo- 
Gricean Studies in Pragmatics and Semantics in Honor of Laurence R. Horn, 1-20. Philadelphia: John Benjamins.

Abrusán, Márta. 2014. Weak Island Semantics. Oxford University Press.

Abusch, Dorit. 2002. Lexical alternatives as a source of pragmatic presuppositions. In Semantics and Linguistic Theory (SALT) 12, 1-19.

Abusch, Dorit. 2010. Presupposition triggering from alternatives. Journal of Semantics 27. 37-80.

Abenina Adar, Maayan \& Yael Sharvit. 2018. Domain uniformity in questions. Handout of a talk given at Semantics and Linguistic Theory (SALT) 28, MIT.

Alonso-Ovalle, Luis. 2006. Disjunction in Alternative Semantics: University of Massachusetts, Amherst PhD dissertation.

Bartels, Christine. 1999. The Intonation of English Statements and Questions: A Compositional Interpretation. New York: Routledge.

Beaver, David \& Emiel Krahmer. 2001. A partial account of presupposition projection. Journal of Logic, Language and Information 10(2). 147-182.

Biezma, María \& Kyle Rawlins. 2017. Rhetorical questions: Severing asking from questioning. In Semantics and Linguistic Theory (SALT) 27, 302-322.

Bolinger, Dwight. 1957. Interrogative Structures of American English. University of Alabama Press, Birmingham.

Caponigro, Ivano \& Jon Sprouse. 2007. Rhetorical questions as questions. In Sinn und Bedeutung (SuB) 11, 121-133.

Champollion, Lucas, Ivano Ciardelli \& Floris Roelofsen. 2017. On questions and presuppositions in typed inquisitive semantics. Talk given at Inquisitiveness Below and Beyond the Sentence Boundary 2.

Chemla, Emmanuel. 2009. Presuppositions of quantified sentences: Experimental data. Natural Language Semantics 17(4). 299-340.

Ciardelli, Ivano, Jeroen Groenendijk \& Floris Roelofsen. 2018. Inquisitive Semantics. Oxford University Press.

Creemers, Ava, Jérémy Zehr \& Florian Schwarz. 2018. Interpreting presuppositions in the scope of quantifiers: every vs. at least one. In Sinn und Bedeutung (SuB) 22, 331-348.

von Fintel, Kai. 2008. What is presupposition accommodation, again? Philosophical Perspectives 22. 137-170.

Gajewski, Jon. 2011. Licensing Strong NPIs. Natural Language Semantics 19(2). 109-148.

Geurts, Bart \& Bob van Tiel. 2016. When "all the five circles" are four: new exercises in domain restriction. Topoi 1(35). 109-122.

Giannakidou, Anastasia. 2007. The landscape of Even. Natural Language \& Linguistic Theory 25(1). 39-81.

Glanzberg, Michael. 2005. Presuppositions, truth values, and expressing proposi- 
An epistemic bridge for presupposition projection in questions

tions. In Gerhard Preyer \& Georg Peter (eds.), Contextualism in Philosophy: Knowledge, Truth, and Meaning, 349-396. Oxford University Press.

Guerzoni, Elena. 2003. Why Even Ask?: On the Pragmatics of Questions and the Semantics of Answers: Massachusetts Institute of Technology $\mathrm{PhD}$ dissertation. Heim, Irene. 1983. On the projection problem for presuppositions. In Michael Barlow, Daniel Flickinger \& Michael Wescoat (eds.), West Coast Conference on Formal Linguistics (WCCFL) 2, 114-125.

Karttunen, Frances \& Lauri Karttunen. 1977. Even questions. In North East Linguistic Society (NELS) 7, 115-134.

Karttunen, Lauri \& Stanley Peters. 1979. Conventional implicature in Montague Grammar. Syntax and Semantics 11. 1-56.

Klinedinst, Nathan. 2016. Two types of semantic presuppositions. In Pragmemes and Theories of Language Use, 601-624. Springer.

Kratzer, Angelika \& Junko Shimoyama. 2002. Indeterminate Pronouns: The View from Japanese. In Y. Otsu (ed.), Tokyo Conference on Psycholinguistics 3, 125.

Nicolae, Andreea C. 2015. Questions with NPIs. Natural Language Semantics 23(1). 21-76.

Pope, Emily. 1976. Questions and Answers in English. The Hague: Mouton.

Rando, Emily. 1980. Intonation in Discourse. In Linda Waugh \& Cornelis Hendrik van Schooneveld (eds.), The Melody of Language, 243-278. Baltimore: University Park Press.

Rooth, Mats. 1985. Association with Focus: University of Massachusetts, Amherst $\mathrm{PhD}$ dissertation.

Rullmann, Hotze. 1997. Even, polarity, and scope. Papers in Experimental and Theoretical Linguistics 4(40-64).

Schlenker, Philippe. 2008. Be articulate: A pragmatic theory of presupposition projection. Theoretical Linguistics 34(3). 157-212.

Schwarz, Bernhard \& Alexandra Simonenko. 2018. Decomposing universal projection in questions. In Sinn und Bedeutung (SuB) 22, 361-374.

Schwarz, Florian, Kajsa Djärv \& Jérémy Zehr. 2018. Do Italian factives entail their presuppositions? Yes, but... In Ilaria Frana Rajesh Bhatt \& Paula Menendez Benito (eds.), Festschrift for Angelika Kratzer, ScholarWorks at UMass.

Simons, Mandy. 2005. Dividing things up: The semantics of or and the modal/or interaction. Natural Language Semantics 13(3). 271-316.

Stalnaker, Robert. 1974. Pragmatic presuppositions. In Milton Munitz \& Peter Unger (eds.), Semantics and Philosophy, 197-214. New York: New York University Press.

Sudo, Yasutada. 2012. On the Semantics of Phi Features on Pronouns: Massachusetts Institute of Technology $\mathrm{PhD}$ dissertation. 
Wilkinson, Karina. 1996. The scope of even. Natural Language Semantics 4(3). 193-215.

Yablo, Stephen. 2006. Non-catastrophic presupposition failure. In Judith Thomson \& Alex Byrne (eds.), Content and Modality: Themes from the Philosophy of Robert Stalnaker, 164-190. Oxford University Press.

Zehr, Jérémy, Cory Bill, Lyn Tieu, Jacopo Romoli \& Florian Schwarz. 2016. Presupposition projection from the scope of None: Universal, existential, or both? In Semantics and Linguistic Theory, vol. 26, 754-774.

Zehr, Jérémy \& Florian Schwarz. 2016. Entailed vs. non-entailed presuppositionsan experimental assessment. In North East Linguistic Society (NELS) 46, 319 328.

Zehr, Jérémy \& Florian Schwarz. 2018. Returning to non-entailed presuppositions again. In Sinn und Bedeutung (SuB) 22, 463-480.

Nadine Theiler

Oak Hall 355

365 Fairfield Way

Storrs, CT

nadine.theiler@uconn.edu 